\title{
ICF-Based Disability Survey in a Rural Population of Adults and Older Adults Living in Cinco Villas, Northeastern Spain: Design, Methods and Population Characteristics
}

\author{
Jesús de Pedro-Cuesta ${ }^{a}$ b Magdalena Comín Comín ${ }^{c} \quad$ Javier Virués-Ortega ${ }^{b}$ \\ Javier Almazán Isla ${ }^{a, b}$ Fuencisla Avellanal ${ }^{a}$ b Enrique Alcalde Cabero ${ }^{a}$ Olga Burzaco ${ }^{d}$ \\ Juan Manuel Castellote ${ }^{e}$ Alarcos Cieza $^{f}$ Javier Damián $^{a}$ b Maria João Forjaz $^{b, g}$ Belén Frades $^{b}$ \\ Esther Franco $^{d}$ Luis Alberto Larrosa ${ }^{\mathrm{h}}$ Rosa Magallón ${ }^{\mathrm{i}}$ Gloria Martín García ${ }^{\mathrm{i}}$ \\ Cristina Martínez $^{d}$ Pablo Martínez Martín ${ }^{\text {b,j }} \quad$ Roberto Pastor-Barriuso ${ }^{a}$ Ana Peña Jiménez ${ }^{i}$ \\ Adolfo Población Martínezk Geoffrey Reed' Cristina Ruíz ${ }^{\mathrm{a}}$ \\ on behalf of the DISCAPARAGON study group \\ ${ }^{a}$ Department of Applied Epidemiology, National Centre for Epidemiology, and ${ }^{b}$ Centro en Red de Investigaciones \\ Biomédicas en Enfermedades Neurodegenerativas (CIBERNED), Carlos III Institute of Health, Madrid, 'Zaragoza

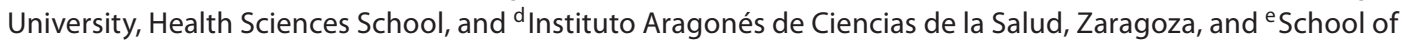

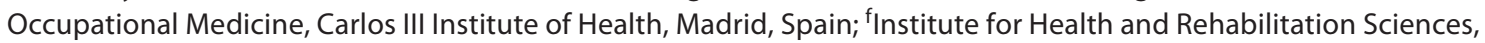 \\ Unit for Biopsychosocial Health, Ludwig Maximilians University, Munich, Germany; ${ }^{9}$ National School of Public Health, \\ Carlos III Institute of Health, Madrid, hDepartment of Family and Social Services, Aragón Government, and \\ 'Research Unit, Centro de Salud Arrabal, Zaragoza, 'Alzheimer Disease Research Unit, Carlos III Institute of Health, \\ Madrid, kFundación Farasdues, Zaragoza, and 'International Union of Psychological Science, Madrid, Spain
}

\section{Key Words}

Chronic diseases $\cdot$ Disability $\cdot$ Dementia $\cdot$ Screening methods · Survey

\begin{abstract}
Background: This article describes the methods of a door-todoor screening survey exploring the distribution of disability and its major determinants in northeastern Spain. This study will set the basis for the development of disability-related services for the rural elderly in northeastern Spain. Methods: The probabilistic sample was composed of 1,354 de facto residents from a population of 12,784 Social Security card holders (age: $\geq 50$ years). Cognitive and disability screenings were conducted (period: June 2008-June 2009). Screening instruments were the MMSE and the World Health Organization
\end{abstract}

Disability Assessment Schedule. Participants screened positive for disability underwent an assessment protocol focusing on primary care diagnoses, disability, lifestyle, and social and health service usage. Participants screened positive for cognitive functioning went through in-depth neurological evaluation. Results: The study sample is described. Usable data were available for 1,216 participants. A total of 625 individuals (51.4\%) scored within the positive range in the disability screening, while 135 (11.1\%) scored within the positive range of the cognitive screening. The proportion of positively screened individuals was higher for women and increased with age. Conclusions: Screening surveys represent a feasible design for examining the distribution of disability and its determinants among the elderly. Data quality may benefit from methodological developments tailored to rural populations with a low education level.

Copyright $\odot 2010$ S. Karger AG, Basel

Jesús de Pedro Cuesta

Centro Nacional de Epidemiología, Instituto de Salud Carlos III

Avenida Monforte de Lemos, 5

ES-28029 Madrid (Spain)

Tel. +34918222 650, Fax +34913877 815, E-Mail jpedro@ isciii.es 


\section{Introduction}

Although some of the most relevant disability determinants are of neurological and mental origin (e.g. dementia, depression), little research effort has been devoted to the neuroepidemiology of functioning and disability [1]. For instance, a review of prevalence surveys of Parkinson's disease [2] showed that disability was assessed solely in three studies [3-5] by the methods of Katz et al. [6], Lawton and Brody [7], and the Schwab and England scales [8]. However, a study conducted in Gironde (France) found that Parkinson's disease patients failed to perform basic and instrumental activities of daily living 5 and 14 times more frequently when compared to controls [3]. Therefore, although seldom assessed, disability may be a major concern among extensive populations of neurological patients. The lack of instruments quantifying disability in epidemiological research may contribute to the scarce data on disability that are available.

The biopsychosocial model and the classification system developed by the World Health Organization (WHO) - i.e. the International Classification of Functioning, Disability and Health (ICF) model [9] - suggest that disability is a general construct not confined to disease-specific factors. The ICF incorporates a comprehensive account of personal, social and environmental factors, and therefore it opens new research avenues for the study of disability and its determinants [10].

The ICF has been used to develop disease-specific disability profiles [11]. In addition, the ICF may also be a framework for assisting the identification of targets in rehabilitation, assessment of intervention outcomes, and social and health service planning [12]. The WHO has also presented several approaches to measure disability consistent with the ICF model, specifically the WHO Disability Assessment Schedule (WHO DAS-II) for epidemiological use [13], and the ICF checklist for clinical use [14]. However, population-based studies targeting elderly participants under one or more health conditions, and neuroepidemiological surveys applying these methods, are absent in the literature.

The ICF Core Sets project (http:www.icf-researchbranch.org/research) represents an attempt to generate disease- and situation-specific practical tools based on the ICF framework [15]. In addition to specific Core Sets, a generic Core Set is also being developed [16]. A generic Core Set should incorporate assessment areas relevant to extensive populations (e.g. rural elderly), and therefore methodological methods will be used in its development. Population-based studies may be an adequate approach to the development of a generic ICF Core Set. On another note, the ICF checklist is designed to allow the eventual inclusion of items not present in the standard version (120 items in the ICF checklist vs. 1,334 items in the complete ICF manual). This flexibility may be necessary in the process of developing a generic Core Set, which needs to cover a diverse population under the potential influence of a number of disability determinants.

The Spanish Act on the Promotion of Personal Autonomy and Care for Dependent Persons enacted in 2006 raised the need of systematic descriptive information on the epidemiology of disability. An ICF-based approach may demonstrate to be superior to that of earlier disability surveys relying solely on self-reported data, as was the case for the Survey on Disabilities, Impairments and Health Status 1999 [17]. Therefore, a population-based study was designed incorporating assessment methods within the ICF framework. The goals of this article are: (1) to present the design, sampling procedure and assessment methods of a population-based survey of people aged 50 years and over living in rural areas in the Cinco Villas county (northeastern Spain), and (2) to describe the geographical and sociodemographic characteristics of the target sample and the performance of the screening procedure. On the other hand, the empirical goals of this project (to be reported elsewhere) were: (1) to describe the prevalence of mild, moderate and severe disability in a rural and semirural population in Aragón (northeastern Spain), (2) to identify risk factors for prevalent disability, particularly diagnoses found systematically in primary care, and (3) to describe selected health-promoting habits and the use of rehabilitation and social support services.

\section{Methods}

It should be noted that disability in Spanish elderly may be accounted for, to a substantial extent, by undiagnosed neurological disorders including dementia, stroke and parkinsonism [18]. In order to capture highly relevant disability determinants among the elderly, the participants were double screened for disability and cognitive function. Screening tests preceded any further assessment. Positively screened participants underwent a comprehensive assessment protocol incorporating the following areas: disability, lifestyle, medical conditions, and use of social, health and rehabilitation services. In addition, participants scoring low in cognitive functioning were submitted to neurological workup.

The participants were sampled from the primary care registry of Social Security card holders. Approaching the target population through the Social Security network has specific benefits: (1) access to medical diagnoses, and (2) external validity of the study. These factors may allow the identification of disease-related disability factors and capitalize on the central role of primary care in 
managing and providing social and rehabilitation services. It should be noted that most Spanish residents have free access to public medical care.

\section{Survey Design}

This study is a population-based prevalence survey of adult participants living in rural areas of the Cinco Villas county. The population was defined as de facto residents of Cinco Villas county holding a Social Security card and born before June 15, 1958. All participants had been official residents during the year preceding the first scheduled visit or more.

\section{Study Population}

The Cinco Villas county is composed of 48 municipalities located in the province of Zaragoza and the region of Aragón (northeastern Spain) (fig. 1). The region was selected due to the logistic support provided by local authorities and nongovernmental organizations. The total population aged 50 years or more in 2008 was 13,315 (Spanish National Institute of Statistics; www. ine.es). The economic wealth in the area pertains to the production and distribution of agricultural goods, services and small industries (Spanish National Institute of Statistics). The region is composed of 2 main geographical areas: southern flat lowlands and northern highlands neighboring the Pyrenees.

\section{Population Sample}

A bona fide sample of 2,000 individuals was randomly selected via their Social Security card number. The registry of Social Security card numbers for the Cinco Villas county was made available by the regional health care statistics unit. Addresses of the participants in the registry were confirmed on a preassessment visit. For the purposes of describing the study population regardless of screening results, a 200-subject subsample was selected randomly from the bona fide sample to undergo full diagnostic and functional evaluation.

\section{Medical and Social Services}

Some $98 \%$ of the population of Aragón are covered by the public health system. Public primary care and mental health services are offered in 5 centers throughout the county. Acute hospital care and specialized medical services are provided in a public hospital in Ejea (fig. 1) and also in Zaragoza city (70-100 km away). A hospital for chronic mental patients is located in Sádaba. In addition, there were 700 residential beds for the elderly in 2007 (Gobierno de Aragón; www.aragon.es). Each individual holding a Social Security card is entitled to claim free health services and subsidized medicine. Health services are expected to develop substantially in the near future due to the implementation of the Act on the Promotion of Personal Autonomy and Care for Dependent Persons.

The assessment was arranged sequentially in 2 steps: screening (phase 1), and diagnosis and functional evaluation (phase 2). The complete de facto population was administered cognitive and disability screening instruments during phase 1 , while in-depth assessments of medical diagnoses, cognition and disability were restricted to those with a positive score in either cognitive or disability screenings.

\section{Assessment Protocol and Instruments}

A comprehensive assessment of health and disability was implemented via 1, 2 or 3 successive home visits by 1 or 2 trained

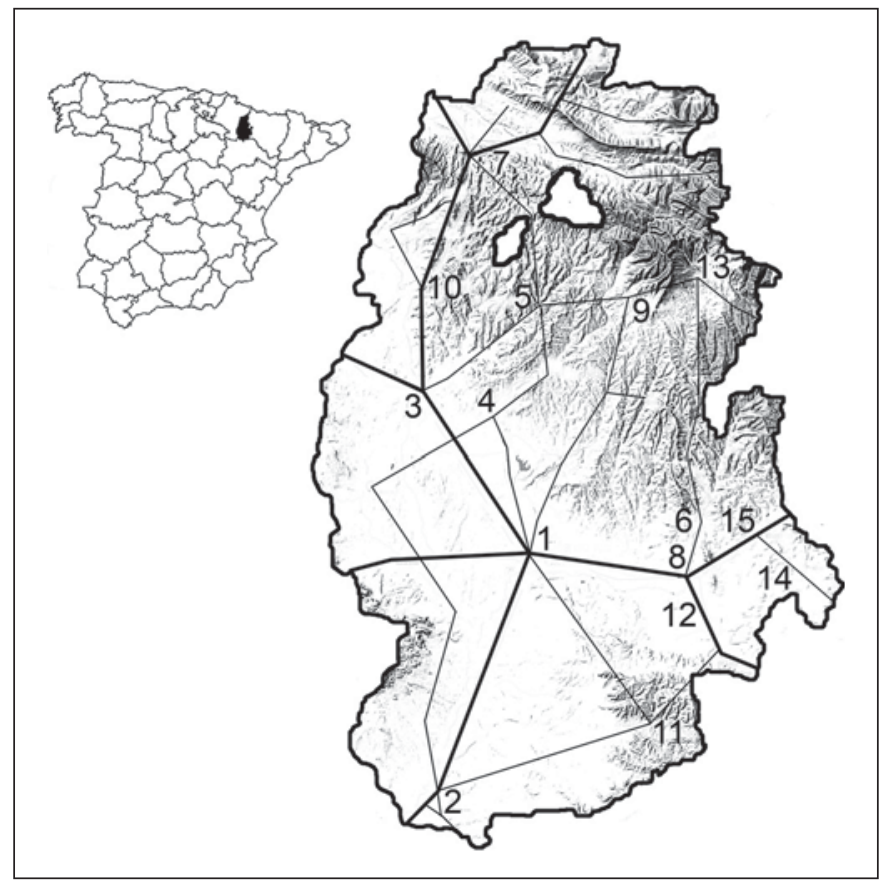

Fig. 1. Geographical location of Cinco Villas county in northeastern Spain. The county map is limited to municipalities with population sizes above 150 inhabitants, the road network and orography. 1 = Ejea (16,249 inhabitants); 2 = Tauste $(7,247) ; 3$ = Sádaba $(1,764) ; 4=$ Biota (1,179); 5 = Uncastillo (875); 6 = Luna (872); 7 = Sos (743); 8 = Erla (445); 9 = Luesia (400); 10 = Castilliscar (384); 11 = Castejón (317); 12 = Sierra de Luna (250); 13 = Biel (213); 14 = Piedratajada (173); 15 = Valpalmas (169). Source: Spanish National Institute of Statistics, 2008.

interviewers. Three assessment protocols on screening, health status and disability were completed at each visit. At the first appointment, informed consent and sociodemographic data were obtained and disability screening instruments administered (MMSE; WHO DAS-II, 12 items; Disability Questionnaire of the Survey on Disabilities, Impairments and Health Status 1999, SDIHS 1999). In the health status protocol, the following areas were explored: physical examination (ad hoc questionnaire), medical history (ad hoc), depression (EURO-D), health-related quality of life (HRQoL; EuroQoL Group 5-Dimension Self-Report Questionnaire, EuroQoL), self-reported physical activity (Yale Physical Activity Scale), consumption of alcohol and tobacco (ad hoc), and preventive practices in health (ad hoc). In the third interview, the following areas were covered: dependence (Katz Extended Index of Dependence in Activities of Daily Living), disability (WHO DAS-II, 36 items; ICF checklist), and use of health and social services (ad hoc). The instruments used for the systematic assessment are described below.

Screening Assessments

Mini-Examen Cognoscitivo/MMSE. The Mini-Examen Cognoscitivo is the Spanish version of the MMSE [19]. Participants scoring below 24 were submitted to neurological workup. 
Disability Questionnaire of the Survey on Disabilities, Impairments and Health Status 1999. The SDIHS 1999 is a semistructured interview for disability screening. This instrument was developed for the Survey on Disabilities, Impairments and Health Status 1999 [17]. The instrument covers 10 areas informed by 3-5 yes/no questions (total: 36 questions). The areas covered are: sight; hearing; communication (language, signs, gestures, writing and reading); learning and performance (recognize objects and persons, time and space orientation, remembering information, perform simple and complex orders); moving around the home; use of arms and hands; transfer outside the home or institution; selfcare; household duties, and interaction with others. The questionnaire's total score is computed as the sum of all items marked as 'yes' (1 point; maximum total: 36 ).

World Health Organization Disability Assessment Schedule II. This is a 12-item questionnaire covering 6 areas of disability referring to the 30-day period before assessment: participation in society; life activities; getting along with others; self-care; getting around, and understanding and communication. Items are answered on a 5-point Likert scale from no (0) to extreme (4) selfreported difficulty in performing a given activity. The 12 -item version has recently been validated for the Spanish population, showing optimal internal consistency (Cronbach's $\alpha=0.89$ ) [13]. The direct score ranges from 0 to 48, with higher scores indicating greater disability. All participants scoring above 0 were administered the complete health and disability protocols (phase 2) including the 36-item version of this instrument [20]. The score per domain and total questionnaire score range from 0 to 100 in the extended version. The 'life activities' domain was omitted for those individuals with no household duties assigned. Items on work performance were also omitted as most participants were retired.

Diagnostic and Functional Evaluation

EURO-D. This is a 12 -item scale assessing the emotional and physical features of depression. The instrument has been validated for elderly respondents including the Spanish population [21, 22]. Its internal consistency is above 0.70 and therefore suited for research use [23]. When administered to the elderly, it has shown significant associations with functional disability $(\beta=0.16-0.37)$, presence of chronic diseases $(\beta=0.08-0.20)$ and sense of coherence $(r=-0.36)[24,25]$.

Yale Physical Activity Scale. This is a semistructured interview on the number of hours devoted to various daily activities (work, yard work, home caretaking, exercise and leisure) during the last 7 days. The scale provides the following indices: total time summary index and energy expenditure summary index. The total time summary index estimates the time involved in physical activities in hours per week. The energy expenditure summary index is an estimation of weekly energy expenditure calculated in kilocalories per hour according to factor weightings associated to each activity [24]. The scale has been validated for the Spanish population [26], has shown an adequate test-retest reliability $(r=$ $0.31-0.65)$ and correlates highly with various physiological indices [26-28].

Katz Extended Index of Dependence in Activities of Daily Living. This is an 11-item scale measuring dependence-based functional status. It surveys 6 basic personal activities of daily living (bathing, dressing, toileting, transferring, continence and feeding) and 5 instrumental activities of daily living (shopping, clean- ing, transportation, washing and cooking) $[6,7]$. The items are rated on a 3-point scale: independent, partly dependent and dependent. Individuals were scored on an 11-point cumulative scale according to Spector et al. [29].

World Health Organization Disability Assessment Schedule II, 36 Items. This has been described above.

EuroQoL Group 5-Dimension Self-Report Questionnaire. This is a descriptive system of 5 dimensions (mobility, usual activities, personal care, pain/discomfort and anxiety/depression) in addition to a visual analog scale of HRQoL. The dimensions of the descriptive system are rated as: no problems, some problems or moderate, and extreme problems or unable. There are 243 possible combinations of responses composing 243 possible health states. A summary index per subject over a $0-1$ scale for each dimension was obtained via the time tradeoff evaluation technique for the Spanish population [30]. The EuroQoL has been used and validated extensively in Spanish populations [30-32].

ICF Checklist Version 2.1A. This is a semistructured schedule to elicit and record information on the functioning and disability of an individual following the major categories of the ICF of the WHO $[9,14]$. The categories explored by the ICF checklist are: impairments of body functions, impairment of body structures, activity limitations and participation restriction, and environmental factors. A distinctive code is used for each condition identified. The ICF rater determines the level of capacity and performance in activities and participation, uses specific qualifiers (level, nature and location of the impairment in body structures and functions), and establishes the extent to which environmental factors function as barriers or facilitators. While nature and location are qualitative qualifiers, impairment, capacity, performance and barrier/facilitator vary over a 0 - to 100 -point scale according to the difficulty experienced by the individual in each condition or activity $(0=$ none; $100=$ complete) [14]. The output of the scale is a list of coded ICF domains with their accompanying qualifiers. The distinction between capacity and performance is a major feature of the ICF assessment schedule. These constructs are indicative of the environment in which a measurement is taking place. According to the ICF, 'performance describes what an individual does in his or her current environment', while 'capacity describes an individual's ability to execute a task or an action and [aims] to indicate the highest probable level of functioning that a person may reach in a given domain at a given moment' $[9, \mathrm{p}$. 15]. In other words, capacity equals performance neutralizing the influence of the environment, and therefore the gap between the two provides an indication as to what can be done to the environment to improve the subject's performance. This is the first study to use the ICF checklist in a general population of rural adults and elderly. Therefore, a consensus was formed over the following areas: specific uses of impairment; nature and location as qualifiers; daily life components of several domains of activities and participation, and definition of a neutral environment for several domains of activities and participation. In addition, the following ICF items were added to the checklist for a better adjustment to the target population: $\mathrm{d} 4600$ - moving around within the home; d5300 - regulating urination; d5301 - regulating defecation; d5408 - dressing, and d5702 - maintaining one's health [9]. Performance, for example in eating and drinking, was scored taking into account regular access to personal help from relatives. A complete listing of the procedural consensus is available upon request. 
Short Version of the Zarit Burden Interview. This is a 12-item structured interview on the burden experienced by caregivers of disabled persons $[33,34]$. Items are answered on a 5-point Likert scale according to how frequently the caregiver believes to be or feels in agreement with the statement of the item $(0-4=$ never to almost always). The total score is computed summing each item's score (range: $0-48$ ). This version has demonstrated optimal internal consistency (Cronbach's $\alpha=0.85$ ). It is also as predictive of mood symptoms as the complete version [35].

Physical Examination. Anthropometrics (weight, height) and arterial pressure readings were obtained by a licensed nurse or primary care physician using a portable tensiometer (Medel Check; ref. No. 90488).

Medical History. The medical history included a checklist of 51 diseases prevalent in the elderly. Extra space was allowed for additional conditions (ICD-10 codes were specified). Primary sources of information were the digital medical file, medical documents held by the examinees, and examinees' self- and proxy reports. This section was administered by a licensed nurse, therapist or primary care physician and was later supervised by a senior physician. Diagnoses of those at chronic care facilities, nursing homes and psychiatric hospitals were informed by medical records and medical practitioners at those sites. Neurological diagnosis was conducted by a licensed neurologist. Diagnoses of dementia and dementia subtypes were conducted by 2 independent licensed neurologists. Disagreements were settled by consensus on a panel of 3 neurologists.

Consumption of Tobacco and Alcohol. Included smoking status (daily smoker, less-than-daily smoker, nonsmoker; number of cigarettes) and smoking history (started when, gave up when, smokefree periods if any). The number of units and frequency of intake of alcoholic beverages over the last year were explored for 5 categories of beverages graded by their alcohol concentration (from beer, wine, champagne and the like up to whisky, rum and the like).

Health Prevention Practices. In order to explore the participants' health responsibility, major health practices in primary care were screened, including: influenza and tetanus immunization over the last year and the last 10 years, respectively; regular blood pressure checks; urine analyses, and sight and hearing examinations. Additionally, female participants were asked about consulting for vaginal cytology and mammography.

Use of Social and Health Services. The participants or relatives were asked about their use of primary care or community-based health services, rehabilitation services, day centers, health teleassistance services, and housekeeping assistance. In addition, the participants were asked about their last hospital admissions, membership in patient associations, and their place of residence (home, nursing home, living with a son or daughter, alone).

\section{Procedure}

Research Team

The research team was composed of specialists from different fields: disability (J.M.C., A.C.), neurology and dementia (P.M.M.), neuroepidemiology (J.P.-C.), psychology (J.V.-O., C.M.), primary care (R.M., G.M.G.), HRQoL (P.M.M., J.F.B.), physical activity (A.P.M., J.M.C.), rehabilitation (M.C.C., J.M.C.), ICF-based research (A.C., G.R.), psychometrics and data analysis (J.V.-O.), physiotherapy (C.M.), occupational therapy (C.R., E.F.), nursing (J.A.I., F.A.), geriatrics (A.P.J.), and statistics (E.A.C.).

\section{Interviewers}

Five assessors with a background as health professionals were selected and trained in the assessment methods described above. The assessors were trained on the administration of the WHO DAS-II by the Spanish WHO Collaborative Center (Marques de Valdecilla Hospital, Santander, Spain). In addition, the interviewers were trained on the ICF checklist by the ICF Research Branch of the Ludwig Maximilians University (Munich, Germany). Coinvestigators provided training to the research assistants in all other assessment methods. Pilot assessments were conducted at a geriatric home before data collection started. An instruction manual for ICF checklist administration focusing on aspects relevant to rural life was built in order to warrant uniform assessment. The interviewers were instructed to verbalize the intent of the item to ensure communication with elderly persons.

Procedure for Individual Assessments

The primary care physicians were informed which of their patients were study participants. Secondly, the participants were informed about the study by e-mail and later reached by telephone up to 4 times until their collaboration status was determined. Initial contacts took place 1 month before the assessment activities were scheduled to begin. Those who accepted were requested permission for 1 or more home visits. The visits and participation status of each subject were also reported to the participants' primary care physicians. The assessment of each individual was divided into the phases described below. All assessments were conducted within the period of June 2008 through June 2009 (prevalence period). Ethical approval was obtained from the Human Subjects Committee of the Aragón Health Sciences Institute.

\section{Study Phases}

All subjects signed an informed consent at their first assessment visit after having accepted to participate in the study. Before data collection started, local primary care physicians and social workers were contacted to facilitate access to the targeted population. In an attempt to improve collaboration, contact with participants was assisted by civil workers and primary care nurses as needed. The study phases are described below.

Screening Phase: Phase 1. The screening protocol was implemented. Information from proxy respondents was requested as needed. If the person was within the $10 \%$ randomly selected reference group, the complete health status and disability assessment protocols were administered at the same visit. Medical findings were confirmed subsequently with the assistance of the participant's primary care physician.

Diagnostic and Functional Evaluation Phase: Phase 2. Individuals screened positively via either the WHO DAS-II, 12 items, or the MMSE, in addition to those randomly selected (10\% bona fide population), participated in a second visit. On a few occasions, a third visit was necessary due to time constraints. Medical reports were examined if the individual was transferred from another area and his/her complete medical record was not available at the local primary care center. Information was also obtained from proxy respondents (relatives and caregivers). Patients scoring above 0 on the WHO DAS-II, 12 items, underwent in-depth disability and health assessments. Patients scoring below 24 on the MMSE were referred to a neurologist for formal diagnosis of dementia and dementia subtype. The diagnostic protocol by Gascón-Bayarri et al. [36] was followed. 


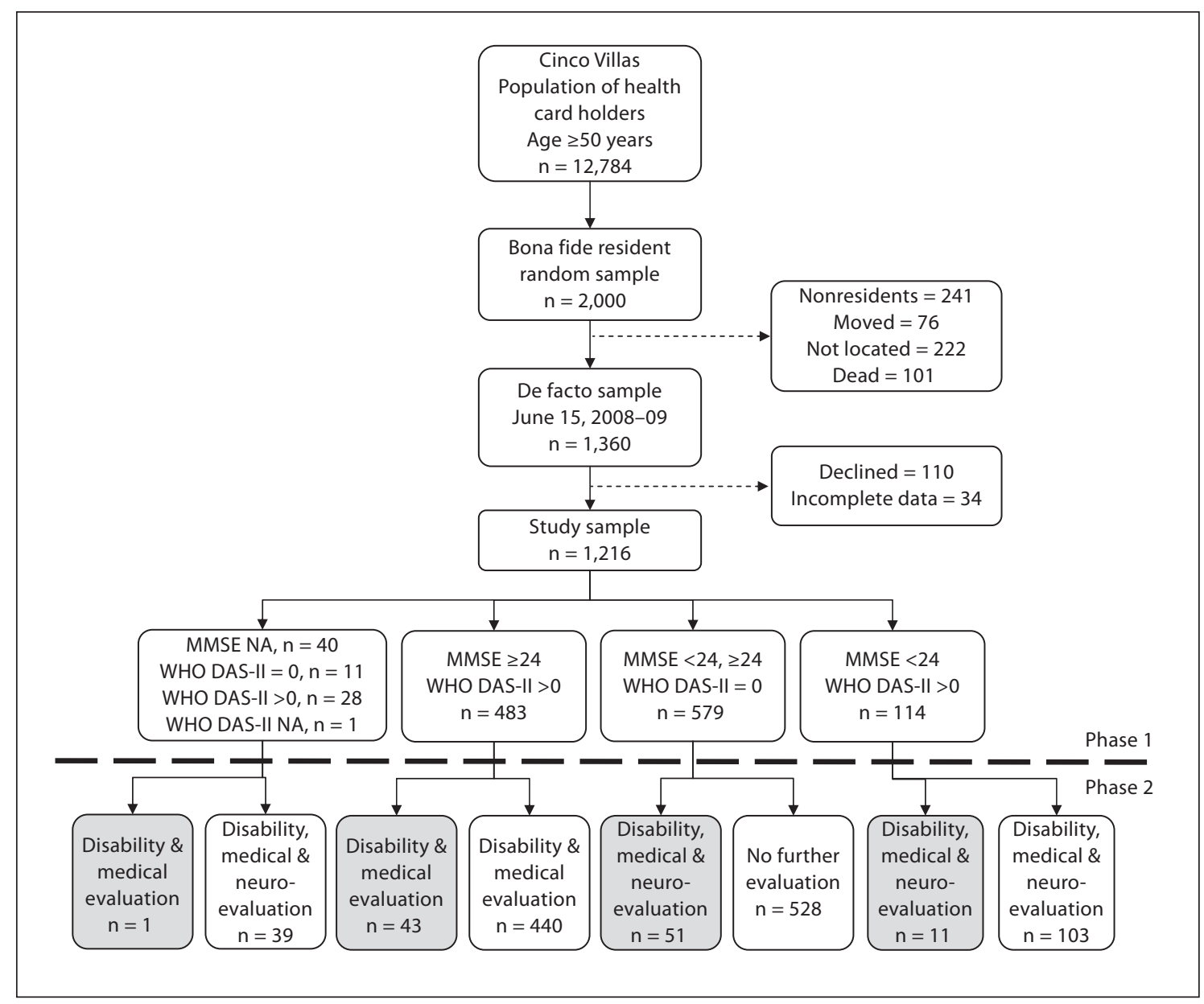

Fig. 2. Flow chart showing the selection of the study sample. Grey boxes: randomly selected $10 \%$ subsample of the bona fide sample $(n=200)$. NA = Not applicable.

\section{Results}

Figure 2 shows the selection flow chart for this survey. In order to reach a bona fide population of 2,000 individuals, simple random sampling (0.156 sampling probability) was implemented over the total population of health card holders (12,784 inhabitants). A total of 646 individuals were excluded from the de facto sample for the following reasons: (1) not actual resident; (2) subject was recently relocated; (3) subject was not found, and (4) subject was dead. The research assistants were not able to confirm the residence of 241 individuals $(12.1 \%$ of the bona fide resident population) either by actual visits to the registered address or by information provided by neighbors. The de facto sample was composed of 1,360 participants. In addition, 110 individuals $(8.1 \%$ of the de facto sample) declined to participate or were unable to schedule or keep assessment appointments. The dataset was incomplete for 34 participants $(2.5 \%$ of the de facto sample).

The final sample was composed of 1,216 individuals with complete assessments (mean age: $69.4 \pm 11.5$ years; $53.4 \%$ women). As shown in table 1 , the number of study participants from each municipality was proportional to the municipality size. Similarly, individuals declining participation were distributed across the municipalities with larger populations (Ejea, Sádaba, Tauste) (table 1).

Table 2 presents the number of individuals scoring above and below the threshold in the cognitive and disability screening tests. According to our results, while cognitive screening is highly selective (135 individuals out of 1,216 ), some form of disability was present in $51.4 \%$ 
Table 1. Population and sample sizes by participating municipalities

\begin{tabular}{|c|c|c|c|c|c|c|}
\hline & \multirow{2}{*}{$\frac{\text { Population }^{1}}{\text { M:F ratio }}$} & \multirow[b]{2}{*}{ total } & \multicolumn{2}{|c|}{ Study sample } & \multicolumn{2}{|c|}{ Declined to participate } \\
\hline & & & $\mathrm{M}: \mathrm{F}$ ratio & total & $\mathrm{M}: \mathrm{F}$ ratio & total \\
\hline Asín & $31: 28$ & 59 & $5: 0$ & 5 & $0: 0$ & 0 \\
\hline Biel & $70: 63$ & 133 & $2: 1$ & 3 & $0: 0$ & 0 \\
\hline Biota & $267: 280$ & 547 & $27: 29$ & 56 & $0: 0$ & 0 \\
\hline Castejón de Valdejasa & $97: 86$ & 183 & $12: 5$ & 17 & $0: 0$ & 0 \\
\hline Castilliscar & $106: 114$ & 220 & $7: 12$ & 19 & $0: 0$ & 0 \\
\hline Ejea de los Caballeros & $2,644: 2,804$ & 5,448 & $246: 288$ & 534 & $50: 15$ & 65 \\
\hline Erla & 103:121 & 224 & $6: 11$ & 17 & $0: 0$ & 0 \\
\hline Frago, El & $39: 30$ & 69 & $1: 1$ & 2 & $0: 0$ & 0 \\
\hline Isuerre & $17: 14$ & 31 & $3: 3$ & 6 & $0: 0$ & 0 \\
\hline Layana & $29: 33$ & 62 & $3: 3$ & 6 & $0: 0$ & 0 \\
\hline Lobera de Onsella & $30: 17$ & 47 & $1: 0$ & 1 & $0: 0$ & 0 \\
\hline Luesia & $118: 108$ & 226 & $10: 9$ & 19 & $0: 0$ & 0 \\
\hline Luna & $242: 225$ & 467 & $22: 20$ & 42 & $4: 0$ & 4 \\
\hline Marracos & $29: 33$ & 62 & $2: 4$ & 6 & $0: 0$ & 0 \\
\hline Navardún & $17: 17$ & 34 & $0: 3$ & 3 & $0: 0$ & 0 \\
\hline Novillas & $161: 158$ & 319 & $15: 13$ & 28 & $0: 0$ & 0 \\
\hline Ores & $40: 33$ & 73 & $5: 0$ & 5 & $0: 0$ & 0 \\
\hline Pedrosas, Las & $29: 32$ & 61 & $2: 3$ & 5 & $0: 0$ & 0 \\
\hline Piedratajada & $32: 47$ & 79 & $3: 2$ & 5 & $0: 0$ & 0 \\
\hline Pintano & $31: 32$ & 63 & $1: 1$ & 2 & $0: 0$ & 0 \\
\hline Pradilla de Ebro & $148: 166$ & 314 & $14: 18$ & 32 & $0: 0$ & 0 \\
\hline Puendeluna & $22: 16$ & 38 & $1: 1$ & 2 & $0: 0$ & 0 \\
\hline Sádaba & $332: 378$ & 710 & $25: 40$ & 65 & $9: 7$ & 16 \\
\hline Sierra de Luna & $70: 84$ & 154 & $7: 8$ & 15 & $0: 0$ & 0 \\
\hline Sos del Rey Católico & $158: 184$ & 342 & $12: 13$ & 25 & $2: 1$ & 3 \\
\hline Tauste & $1,268: 1,462$ & 2,730 & $113: 138$ & 251 & $17: 4$ & 21 \\
\hline Uncastillo & 207:227 & 434 & $17: 15$ & 32 & $0: 0$ & 0 \\
\hline Undués de Lerda & $20: 12$ & 32 & $0: 3$ & 3 & $0: 0$ & 0 \\
\hline Urriés & $33: 21$ & 54 & $2: 2$ & 4 & $0: 0$ & 0 \\
\hline Valpalmas & $42: 58$ & 100 & $2: 4$ & 6 & $0: 0$ & 0 \\
\hline Total & $6,432: 6,883$ & 13,315 & $566: 650$ & 1,216 & $82: 27$ & 109 \\
\hline
\end{tabular}

of the population (625 individuals). The number of individuals screened positively both in the cognitive and the disability screening tests increased proportionally with age. Specifically, the proportion of positively screened individuals in the cognitive test ranged from $3.1 \%$ at the lowest age category to $94.7 \%$ for individuals aged 90 years or more, and from 33.4 to $89.5 \%$ for those screened positive in the disability test (table 2). The MMSE was not applicable to 40 individuals due to refusal to respond, dementia and major disability (fig. 2).

Above $85 \%$ of the participants were married or widowed, lived at their own homes with their families and were of low or mid-to-low social class (table 3). The level of education of the study sample was low, with 7.8 average years of schooling. The modally highest education level was primary. Low education categories (none, incomplete primary and complete primary education) accounted for $86.6 \%$ of the participants. Most individuals were retired at the time of the study (79.4\%). In addition, $20.6 \%$ were working and $21.6 \%$ were devoted to housekeeping. Blue-collar and service professions accounted for $89.9 \%$ of the working background of the participants. Additional features of the study participants are available from table 3. 
Table 2. Study sample distribution (n) of screening outcomes by

\begin{tabular}{|c|c|c|c|c|c|c|c|}
\hline & \multicolumn{4}{|c|}{ Sample MMSE screening } & \multicolumn{3}{|c|}{$\begin{array}{l}\text { WHO DAS-II } 12 \\
\text { items screening }\end{array}$} \\
\hline & & negativ & positive & NA & negative & positive & NA \\
\hline \multicolumn{8}{|l|}{ Men } \\
\hline $50-59$ years & 142 & 132 & 6 & 4 & 104 & 38 & 1 \\
\hline $60-69$ years & 154 & 149 & 2 & 3 & 114 & 39 & 0 \\
\hline $70-79$ years & 173 & 150 & 14 & 9 & 93 & 80 & 0 \\
\hline $80-89$ years & 86 & 58 & 25 & 3 & 23 & 63 & 0 \\
\hline$\geq 90$ years & 11 & 5 & 4 & 2 & 2 & 9 & 0 \\
\hline Total & 566 & 494 & 51 & 21 & 336 & 229 & 1 \\
\hline \multicolumn{8}{|l|}{ Women } \\
\hline $50-59$ years & 157 & 154 & 3 & 0 & 95 & 62 & 0 \\
\hline $60-69$ years & 151 & 148 & 2 & 1 & 74 & 77 & 0 \\
\hline $70-79$ years & 179 & 152 & 22 & 5 & 59 & 120 & 0 \\
\hline $80-89$ years & 137 & 86 & 43 & 8 & 25 & 112 & 0 \\
\hline$\geq 90$ years & 26 & 7 & 14 & 5 & 1 & 25 & 0 \\
\hline Total & 650 & 547 & 84 & 19 & 254 & 396 & 0 \\
\hline \multicolumn{8}{|l|}{ Both } \\
\hline $50-59$ years & 299 & 286 & 9 & 4 & 199 & 100 & 1 \\
\hline $60-69$ years & 305 & 297 & 4 & 4 & 188 & 116 & 0 \\
\hline $70-79$ years & 352 & 302 & 36 & 14 & 152 & 200 & 0 \\
\hline $80-89$ years & 223 & 144 & 68 & 11 & 48 & 175 & 0 \\
\hline$\geq 90$ years & 37 & 12 & 18 & 7 & 3 & 34 & 0 \\
\hline Total & $\overline{1,216}$ & 1,041 & 135 & 40 & 590 & 625 & 1 \\
\hline
\end{tabular}

NA $=$ Not applicable age groups and $\operatorname{sex}(n=1,216)$

Table 3. Characteristics of the study sample $(n=1,216)$

Sociodemographic

Age, years

Gender (M:F), n (\%)

$69.43 \pm 11.5$

Marital status, \%

Married or lives with partner $\quad 67.9$

Widow/widower 20.6

Divorced or separated $\quad 1.1$

Single $\quad 10.3$

Living arrangements, \%

Alone

12.9

With others

87.1

Residential status, \%

Own home $\quad 94.3$

Institutionalized $\quad 5.7$

Social class $^{1}, \%$

Low $\quad 37.6$

Mid-to-low $\quad 49.7$

Average $\quad 10.4$

Mid-to-high $\quad 1.6$

High 0.7

\begin{tabular}{lr}
\hline Anthropometric & \\
Height, cm & $160.9 \pm 10.1$ \\
Weight, kg & $73.8 \pm 15.2$ \\
Body mass index & $29.6 \pm 18.7$ \\
Blood pressure ${ }^{2}, \mathrm{~mm} \mathrm{Hg}$ & $96.7 \pm 10.2$ \\
\hline Education and work & \\
Total years of schooling & $7.8 \pm 4.0$ \\
Highest academic degree, \% & \\
$\quad$ None, incomplete primary education & 43.1 \\
$\quad$ Primary education & 43.5 \\
$\quad$ Higher than primary education & 13.4 \\
Current work status, \% & \\
$\quad$ Not working, retired & 55.7 \\
$\quad$ Not working, government subsidized & 2.1 \\
$\quad$ Not working, housekeeping & 21.6 \\
$\quad$ Working & 20.6 \\
Work history, \% & \\
$\quad$ Blue-collar & 56.8 \\
$\quad$ White-collar & 10.1 \\
$\quad$ Services & 33.1 \\
\hline
\end{tabular}

To our knowledge, this study is the first population survey implementing a simultaneous double screening for disability and cognition, which also incorporates a comprehensive assessment and diagnostic phase with complete access to primary care records. ICF checklist disability assessment in addition to the evaluation of clinical, environmental and personal factors may be the basis for health and social service planning in a given population. This study illustrates this process in a particularly complex rural population with prevalent chronic neurological diseases.

Health card registry was assumed to be a comprehensive listing of subjects in the population. However, it became apparent that the registry of health card holders was to some extent outdated. A high number of nonactual residents may be attributed to temporary immigrant residents and individuals having transferred to urban areas without notice.

\section{Environment}

Housing standards (range: $0-17)^{3}$

Median access to community services (range: $1-5)^{4}$

Primary care

Public transportation

Public parks

Shops

Social clubs

Results are expressed as means $\pm \mathrm{SD}$, unless otherwise specified

${ }^{1}$ As estimated by education level and work history according to the algorithm by Rojo et al. [37]. ${ }^{2}$ Mean arterial pressure $=$ diastolic blood pressure $+0.33 \times$ (systolic blood pressure - diastolic blood pressure). ${ }^{3}$ Housing standards were measured by the presence/absence of: air conditioning, car, computer, running water, dish washer, elevator, flush toilet, fridge, garden, gate keeper, heating system, hot water, parking space, telephone, shower or bathtub, and television. ${ }^{4}$ Median and rank of self-reported scale $(1=$ very easy; 5 = very hard). 
A considerable number of persons were never located. The former or current residence of these persons was neither affirmed nor denied by telephone, personal visit or requests of information from neighbors. It is possible that healthy males of working age constituted a substantial proportion of the nonlocated residents. Specifically, it is highly likely that sampling may have been biased against nondisabled 50- to 59-year-old male participants as indicated by the even number of positively screened individuals across the 50- to 59-year and 60 - to 69-year age categories. Similarly, more limited participation in men living in Ejea and Tauste during the harvest season may suggest the same.

Interviewers were entitled to provide further clarification remarks beyond the literal composition of the items in order to make sure that the examinee understood the intent of the items. However, a number of participants experienced difficulties in understanding some of the items, according to the interviewers' report, due to the low average education level of the study sample and, occasionally, due to localisms that prevented fluent communication between interviewer and examinee. This was particularly evident for the WHO DAS-II, 12 items, which showed a number of nonapplicable (not understood) items. However, this had no impact on the actual performance of the screening process as most individuals with nonapplicable items had 1 or more items with some degree of disability. However, the few nonapplicable screening tests (40 MMSE, 1 WHO DAS-II) were caused by specific disabilities (dementia, aphasia).

Sensitivity is an important determinant of measurement quality. The validity of the information obtained for screening may be poorly known, both for the MMSE and WHO DAS-II. MMSE sensitivity for dementia was $89.9 \%$ in the original Spanish validation [19]. Evidence of reduced MMSE sensitivity in individuals with higher educational levels has prompted some authors to suggest a cutoff adjustment in accordance with educational level [38]. However, this adjustment has not been explored for the Spanish version of this instrument. Data from the randomly selected subsample may prove useful for further sensitivity analysis of this instrument. With regard to the Spanish version of the WHO DAS-II [20], its sensitivity for detecting different levels of disability is not known. Unlike the MMSE, there is no reference criterion for disability in order to test sensitivity. However, our study will be the basis of convergent and known-group validity analyses that will inform the psychometric performance of the WHO DAS-II as a screening tool [39]. In addition, the implementation of dementia diagnosis in the future analysis of this dataset will make it possible to provide data on false-positive cases in the screening. False negatives for the disability screening may be identified through the analysis of the $10 \%$ random subsample drawn from the bona fide population.

The development of a methodological armamentarium for the assessment of disability and HRQoL is a major issue in clinical and general neuroepidemiology. However, unlike other disciplines [40], instruments measuring disability and HRQoL have been heavily influenced by clinical profile with little attention to behavioral, social and environmental factors [41]. The ICF model may be a promising framework for bridging this gap. Specifically the choice of assessment instruments in this project has the potential to evaluate the performance of disability and HRQoL assessments using specific behavioral, social and environmental factors as independent variables. In this respect, the ICF checklist may provide valuable guidance in the form of specific variables and clusters that an individual or a population presents. This approach may expand the epidemiological analysis of disability and its complex relation between the individual and his/her environment. For instance, the amount and nature of assistance required by patients with Parkinson's disease and a low Katz index may vary greatly depending on which specific skills are preserved, the availability of a supportive social network, and the impact of the physical environment on the individual's mobility.

Disease-specific ICF Core Sets have been found a useful methodological strategy, e.g. to assess the rehabilitation of patients with stroke [42]. In addition, high comorbidity in elderly populations suggests that a generic Core Set needs to be developed. The expansion of some items of the ICF checklist potentially useful for building a generic Core Set is a long-term goal of this project.

In summary, we have presented the design, procedure and methods as well as the study sample characteristics of a population survey of disability in middle- and advanced-aged rural residents. The design combined disability screening in addition to specific methods to identify common causes of neurological disability. While this approach was successful, future studies may strengthen the data quality by the adaptation of self-reported measures to be administered to adults and older adults with low education levels living in rural areas. The information comprised in this study may prove useful for planning social and rehabilitation services for rural older adult and elderly populations. 


\section{Acknowledgments}

The authors would like to acknowledge the contribution of Esteban de Manuel Keenoy and Anselmo López (project management; Aragón Health Sciences Institute), Susana Coloma and Mariano Cortés (advertising; Farasdués Foundation), Elena Espes (coordination), Soledad Corbacho (computer support; SALUD), Javier Lambán, Eduardo Alonso and José Luis Pola (city, county and provincial administration support), Heinrich Gall (data entry), Gloria Fernández-Mayoralas and Fermina Rojo (questionnaire design), and the Garcés family (logistic support). Special thanks go to the Aragón Government Departments of Health and Social Affairs for institutional support and to the Arrabal Health Center for their assistance with administrative and logistic tasks. The authors are indebted to the general practitioners, psychiatrists, social workers and the staff of primary care centers and nursing homes in Cinco Villas for their continuous support for the project. Financial support came from the Carlos III Institute of Health (projects EPI 1637/06 and 1530/07; Health Research Fund FIS PI06/1098 and PI07/90206), CIBERNED network, Diputación de Zaragoza and Farasdués Foundation.

This study was conducted by the DISCAPARAGON Research Consortium (individuals and groups below):

- Carlos III Institute of Public Health, Madrid, Spain: J.A.I., F.A., E.A.C., J.M.C., J.D., J.F.B., B.F., P.M.M., J.P.-C. and J.V.-O.

- Zaragoza University, Zaragoza, Spain: M.C.C. and A.P.J.

- Ludwig Maximilians University, Munich, Germany: A.C., Heinrich Gall and G.R.

- Aragón Health Sciences Institute, Zaragoza, Spain: O.B., E.F., C.R. and C.M.

- Aragón Health Service SALUD, Zaragoza, Spain: Natividad Romanos and Maria José Tris.

- Fundación Farasdués, Zaragoza, Spain: A.P.M.

- Research Unit Centro de Salud Arrabal SALUD, Zaragoza, Spain: R.M. and G.M.G.

\section{References}

1 Gutenbrunner C, Ward AB, Chamberlain MA: White book on physical and rehabilitation medicine in Europe. J Rehabil Med Suppl 2007;39:1-48.

2 de Pedro-Cuesta J: Epidemiology of Parkinson disease-related disability: disability assessment in Parkinson's disease (in Spanish); in Martínez-Martin P (ed): Valoración de la discapacidad en la enfermedad de Parkinson. Madrid, Real Patronato sobre Discapacidad, 2002, pp 38-39.

- 3 Tison F, Dartigues JF, Dubes L, Zuber M, Alpérovitch A, Henry P: Prevalence of Parkinson's disease in the elderly: a population study in Gironde, France. Acta Neurol Scand 1994;90:111-115.

-4 Tandberg E, Larsen JP, Nessler EG, Riise T, Aarli JA: The epidemiology of Parkinson's disease in the county of Rogaland, Norway. Mov Disord 1995;10:541-549.

$\checkmark 5$ Schrag A, Ben-Shlomo Y, Quinn NP: Crosssectional prevalence survey of idiopathic Parkinson's disease and parkinsonism in London. BMJ 2000;321:21-22.

$\checkmark 6$ Katz S, Ford AB, Moskowitz RW, Jackson BA, Jaffe MW: Studies of illness in the aged: the index of activities of daily living - a standardized measure of biological and psychosocial function. JAMA 1963;185:914-919.

7 Lawton MP, Brody EM: Assessment of older people: self-maintaining and instrumental activities of daily living. Gerontologist 1969; 9:179-186.

8 Schwab RS, England AC: Projection techniques for evaluation surgery in Parkinson's disease; in Gillingham FJ, Donaldson MC (eds): Third Symposium on Parkinson's Disease. Edinburgh, Royal College of Surgeons, 1968, pp 152-157.
9 World Health Organization: International Classification of Functioning, Disability and Health. Geneva, World Health Organization, 2009

10 Jelsma J: Use of the International Classification of Functioning, Disability and Health: a literature survey. J Rehabil Med 2009;41:1-12.

11 Cieza A, Ewert T, Ustün TB, Chatterji S, Kostanjsek N, Stucki G: Development of ICF Core Sets for patients with chronic conditions. J Rehabil Med 2004;44(suppl 1):9-11.

12 McDaid D, Cieza A, Gomez AR: Bridging knowledge: reflections on crossing the boundaries between long-term care and support. Int J Integr Care 2009;9:e60.

13 Luciano JV, Ayuso-Mateos JL, Fernández A Serrano-Blanco A, Roca M, Haro JM: Psychometric properties of the twelve-item World Health Organization Disability Assessment Schedule II (WHO-DAS II) in Spanish primary care patients with a first major depressive episode. J Affect Disord 2010;121:52-58.

14 World Health Organization: ICF checklist version 2.1A. Geneva, World Health Organization, 2009. http://www.who.int/classifications/icf/training/icfchecklist.pdf.

15 Brockow T, Cieza A, Kuhlow H, Sigl T, Franke T, Harder M, Stucki G: Identifying the concepts contained in outcome measures of clinical trials on musculoskeletal disorders and chronic widespread pain using the International Classification of Functioning, Disability and Health as a reference. J Rehabil Med 2004;44(suppl 1):30-36.

16 Cieza A, Geyh S, Chatterji S, Kostanjsek N, Ustün BT, Stucki G: Identification of candidate categories of the International Classification of Functioning Disability and Health (ICF) for a generic ICF Core Set based on regression modeling. BMC Med Res Methodol 2006;6:36
17 Spanish National Institute of Statistics: Survey on Disabilities, Impairments and Health Status 1999 (in Spanish). Madrid, INE, 2001.

18 Zunzunegui-Pastor MV, del Ser T, Rodríguez-Laso A, García de Yébenes MJ, Domingo J, Otero-Puime A: Undetected dementia and health service use: implications for primary care (in Spanish). Aten Primaria 2003; 31:581-586.

19 Lobo A, Saz P, Marcos G, Día JL, de la Cámara C, Ventura T: Validation and norms of the Mini-Examen Cognoscitivo, the first Spanish version of the MMSE, in the general geriatric population (in Spanish). Med Clin (Barc) 1999;112:767-774.

-20 Vázquez-Barquero JL, Vázquez-Bourgón E, Herrera-Castanedo S, Saiz J, Uriarte M, Morales F: Spanish version of the new World Health Organization Disability Assessment Schedule II (WHO-DAS-II): initial phase of development and pilot study (in Spanish). Actas Esp Psiquiatr 2000;28:77-87.

-21 Prince MJ, Reischies F, Beekman AT, Fuhrer R, Jonker C, Kivela SL, Lawlor BA, Lobo A, Magnusson H, Fichter M, van Oyen H, Roelands M, Skoog I, Turina C, Copeland JR: Development of the EURO-D scale: a European Union initiative to compare symptoms of depression in 14 European centres. Br J Psychiatry 1999;174:330-338.

22 Larraga L, Saz P, Dewey ME, Marcos G, Lobo A: Validation of the Spanish version of the EURO-D scale: an instrument for detecting depression in older people. Int J Geriatr Psychiatry 2006;21:1199-1205.

23 Scientific Advisory Committee of the Medical Outcomes Trust: Assessing health status and quality-of-life instruments: attributes and review criteria. Qual Life Res 2002;11: 193-205. 
-24 Braam AW, Prince MJ, Beekman AT, Delespaul P, Dewey ME, Geerlings SW, Kivela SL, Lawlor BA, Magnusson H, Meller I, Pérès K, Reischies FM, Roelands M, Schoevers RA, Saz P, Skoog I, Turrina C, Versporten A, Copeland JR: Physical health and depressive symptoms in older Europeans: results from EURODEP. Br J Psychiatry 2005; 187:35-42.

-25 Virués-Ortega J, Martínez-Martín P, del Barrio JL, Lozano LM: Cross-cultural validation of Antonovsky's Sense of Coherence Scale (OLQ-13) in Spanish elders aged 70 years or more (in Spanish). Med Clin (Barc) 2007;128:486-492.

26 de Abajo S, Larriba R, Marquez S: Validity and reliability of the Yale Physical Activity Survey in Spanish elderly. J Sports Med Phys Fitness 2001;41:479-485.

-27 DiPietro L, Caspersen A, Ostfeld M, Nadel E: A survey for assessing physical activity among older adults. Med Sci Sports Exerc 1993;25:628-642.

-28 Schuler PB, Richardson MT, Ochoa P, Wang MQ: Accuracy and repeatability of the Yale Physical Activity Survey in assessing physical activity of older adults. Percept Mot Skills 2001;93:163-177.

-29 Spector WD, Katz S, Murphy JB, Fulton JP: The hierarchical relationship between activities of daily living and instrumental activities of daily living. J Chron Dis 1987;40:481489.
30 Badía X, Roset M, Montserrat S, Herdman M, Segura A: The Spanish version of the EuroQoL: description and applications (in Spanish). Med Clin (Barc) 1999;112(suppl 1):79-85.

-31 Casellas F, Vivancos JL, Sampedro M, Malagelada JR: Relevance of the phenotypic characteristics of Crohn's disease in patient perception of health-related quality of life. Am J Gastroenterol 2005;100:2737-2742.

32 Casellas F, Arenas JI, Baudet JS, Fábregas S, García N, Gelabert J, Medina C, Ochotorena I, Papo M, Rodrigo L, Malagelada JR: Impairment of health-related quality of life in patients with inflammatory bowel disease: a Spanish multicenter study. Inflamm Bowel Dis 2005;11:488-496.

33 Zarit S, Orr N, Zarit J: The Hidden Victims of Alzheimer's Disease. Families under Stress. New York, University Press, 1985.

34 Bédard M, Molloy DW, Squire L, Dubois S, Lever JA, O’Donnell M: The Zarit Burden Interview: a new short version and screening version. Gerontologist 2001;41:652-657.

35 O’Rourke N, Tuokko HA: Psychometric properties of an abridged version of the Zarit Burden Interview within a representative Canadian caregiver sample. Gerontologist 2003;43:121-127.
36 Gascón-Bayarri J, Reñé R, del Barrio JL, de Pedro-Cuesta J, Ramon JM, Manubens JM, Sánchez C, Hernández M, Estela J, Juncadella M, Rubio FR: Prevalence of dementia subtypes in El Prat de Llobregat, Catalonia, Spain: the PRATICON Study. Neuroepidemiology 2007;28:224-234.

37 Rojo Perez F, Fernandez-Mayoralas G, Pozo Rivera FE, Rojo Abuin JM: Ageing in place: predictors of the residential satisfaction of elderly. Soc Indic Res 2001;54:173-208.

38 Crum RM, Anthony JC, Bassett SS, Folstein MF: Population-based norms for the MiniMental State Examination by age and educational level. JAMA 1993;269:2386-2391.

39 Netemeyer RG, Bearden WO, Sharma S: Scaling Procedures. Issues and Applications. Thousand Oaks, Sage, 2003.

40 Jette A: Toward a common language of disablement. J Gerontol A Biol Sci Med Sci 2009; 64:1165-1168.

41 de Pedro-Cuesta J, Forjaz J: Neurological diseases, disability and quality of life (in Spanish); in Martínez-Martín P (ed): Calidad de Vida en Neurología. Madrid, Ars XXI, 2005, pp 151-166.

42 Geyh S, Cieza A, Schouten J, Dickson H, Frommelt P, Omar Z, Kostanjsek N, Ring H, Stucki G: ICF Core Sets for stroke. J Rehabil Med 2004;44(suppl 1):135-141. 